\title{
Search for the astrophysical sources of the Fly's Eye event with the highest to date cosmic ray energy $E=3.2 \cdot 10^{20} \mathrm{eV}$
}

\author{
R. B. Gnatyk ${ }^{1 *}$ Yu. N. Kudrya ${ }^{2}$, V.I. Zhdanov ${ }^{2}$ \\ ${ }^{1}$ Faculty of Physics, Taras Shevchenko National University of Kyiv, Glushkova ave., 4, 03127, Kyiv, Ukraine \\ ${ }^{2}$ Astronomical Observatory, Taras Shevchenko National University of Kyiv, Observatorna str., 3, 04053, Kyiv, Ukraine
}

\begin{abstract}
Among the registered extremely high energy cosmic rays (EHECR, $E>10^{20} \mathrm{eV}$ ) an event with the highest to date energy of $E=3.2 \cdot 10^{20} \mathrm{eV}$ was detected by the Fly's Eye experiment (FE event) in 1991 . With the use of the back-tracking method for the calculation of the EHECR trajectories in Galactic and extragalactic magnetic fields, we show that the galaxies UGC 03574 and UGC 03394 are the most promising candidates among the nearby extragalactic sources for the cases of iron and C-N-O group primary nucleus respectively. The most likely accelerating mechanisms are the newly-born millisecond pulsars, magnetar flares and tidal disruption events in these galaxies.
\end{abstract}

Key words: ISM: cosmic rays, magnetic fields; stars: pulsars, magnetars

\section{INTRODUCTION}

The extremely high-energy cosmic rays (EHECR) with the energy $E>10^{20} \mathrm{eV}$ are extremely rare phenomena in the observed CR flux: till now two largest modern detectors registered only six (Pierre Auger Observatory (PAO, AUGER)) and ten (Telescope Array (TA)) such events [1, 2]. Potential sources of EHECR are considered to be among the active galactic nuclei (AGN, especially blazars), cosmological gamma-ray bursts, tidal disruptions of stars in the neighbourhood of the supermassive black holes, newly-born millisecond pulsars and magnetar flares [21]. From the first three classes of objects we expect a certain flux of light nuclei - protons and helium (charge $\mathrm{Z}=1,2$ ), whereas from the neutron stars with an iron crust we can also expect iron nuclei $(\mathrm{Z}=26)$ and their decay products, including the $\mathrm{C}-\mathrm{N}-\mathrm{O}$ group $(\mathrm{Z}=6-8)$. The observed nearly isotropic flux of UHECR with $E>10^{18} \mathrm{eV}$ suggests a significant deviation of UHECR in the Galactic and intergalactic magnetic fields [1, 2]. Therefore we should consider predominantly the EHECR events with the minimal magnetic deflection when searching for the correlation of their arrival directions with the positions of potential sources. Furthermore, the sources of EHECR should be close enough to the Earth due to the GZK cut-off: the GZK horizon for EHECR protons is smaller than $40 \mathrm{Mpc}$ [12]. Similar restrictions exist for the propagation length of the nuclei due to photodisintegration [6]. The most suitable CR event in this respect is the event with the highest detected energy. This event was registered by theFly's Eye (FE) experiment in 1991 with the following parameters: $E=(3.2 \pm 0.9) \cdot 10^{20} \mathrm{eV}$,

\footnotetext{
*roman_hnatyk@ukr.net
}

(C) R. B. Gnatyk, Yu.N. Kudrya, V.I.Zhdanov, 2016
$\mathrm{RA}=(85.2 \pm 0.5)^{\circ}$, Dec $=\left(48.0_{-6.3}^{+5.2}\right)^{\circ}($ the galactic coordinates are centred at $l=163.4^{\circ}$ and $\left.b=9.6^{\circ}\right)$ and the shower maximum $X_{\max }=815 \mathrm{~g} / \mathrm{cm}^{2}[10]$. Unfortunately, the nature of the original particle could not be established, as the observed shower properties can be accommodated with both the proton (QGSJETII-03 shower model in the TA collaboration) and an iron nuclei (EPOS-LHC shower model in the PAO collaboration) [4].

Already in 1995 in [16] it was shown that the FE event posses a serious problem: interaction of a CR particle with the cosmic background radiation restricts its mean free path to less than $50 \mathrm{Mpc}$ (a nucleus with the FE-event energy can not reach the Earth from the distances beyond $10 \mathrm{Mpc}$ [17], the mean free paths of similar energy protons (against photomeson losses) and photons (against electronpositron pair production) are less than $30 \mathrm{Mpc}$ [23]), while the potential astrophysical sources in the sky region around the $\mathrm{CR}$ arrival direction are at considerably larger distances (see also $[5,8]$ ). These difficulties with the astrophysical sources stimulated the development of particle physics (especially beyond the Standard Model) theories of the FE event, namely, EHECR from topological defects, from super heavy dark matter decay etc. [9]. The peculiarity of the decaying top-down models is a photondominated flux [7], however, according to the recent PAO results on the integral flux upper limit of the ultra high-energy photons [11], the top-down models of UHECR are strongly disfavoured.

In this work we search for the astrophysical sources of EHECR inside the FE event horizon of 30$40 \mathrm{Mpc}$ (corresponding to the redshift of $z<0.01$ ), taking into account the favoured transient sources 
of EHECR in the normal galaxies (newly-born millisecond pulsars, magnetar flares, tidal disruption events) [21] and the recent models of the Galactic magnetic field $[19,20]$ to recover the trajectory of the FE cosmic ray and determine the Galactic and extragalactic objects that could be the sources of the FE event.

\section{PROPAGATION IN THE GALACTIC AND}

\section{EXTRAGALACTIC MAGNETIC FIELDS}

On their way from the source to the Earth EHECRs are deflected in the Galactic and extragalactic magnetic fields. Galactic magnetic field can be presented as a sum of regular and random components $[14,19,20]$. Regular magnetic field consists of a spiral disk field with a structure that corresponds to the structure of spiral arms, a toroidal field of the Galactic halo and the X-field - the axisymmetric poloidal field directed from the southern to the northern hemisphere of the Galaxy [20]. The trajectory of a cosmic ray with the energy $E$ and charge $q=e \mathrm{Z}$ in a magnetic field $\mathbf{B}$ is described by the equation of motion for the radius vector $\mathbf{r}$ and velocity $\mathbf{v}$ (for the EHECR Lorentz factor $\gamma \gg 1$ and $|\mathbf{v}| \approx c$, neglecting the energy losses only the direction of the velocity vector is changing):

$$
\frac{d \mathbf{r}}{d t}=\mathbf{v} ; \quad \frac{d \mathbf{v}}{d t}=\frac{q c^{2}}{E}[\mathbf{v} \times \mathbf{B}] .
$$

A reconstruction of the cosmic ray trajectory can be achieved via the method of inverse trajectories: we launch a cosmic ray with the detected energy $E$, but with the inverse charge $-q$, towards the arrival direction of the EHECR from the position of the observer in the Galaxy (here we take the solar system coordinates $x=-8.5 \mathrm{kpc}, y=0.0 \mathrm{kpc}, z=0.02 \mathrm{kpc}$ with respect to the Galactic centre). The calculated in this way trajectory recreates the trajectory of the detected UHECR.

Now let us consider the role of the random components of the Galactic and extragalactic magnetic fields. The extragalactic magnetic field is usually simulated in form of cells with the typical size (coherence length) of $l_{B}=0.1 . .1 \mathrm{Mpc}$ and a random distribution of the magnetic field amplitude $B_{\mathrm{rms}}=$ $10^{-12} . .10^{-9} \mathrm{G}[14,24]$. EHECR with energy $E$ from an extragalactic source at a distance $D$ in this field is deflected at the angle $\theta_{\text {rms }}$ :

$$
\begin{aligned}
\theta_{\mathrm{rms}}(E, D) & \simeq 0.08^{\circ} \cdot Z\left(\frac{E}{10^{20} \mathrm{eV}}\right)^{-1} \times \\
\times & \left(\frac{D}{10 \mathrm{Mpc}}\right)^{\frac{1}{2}}\left(\frac{l}{1 \mathrm{Mpc}}\right)^{\frac{1}{2}}\left(\frac{B_{\mathrm{rms}}}{10^{-10} \mathrm{G}}\right) .
\end{aligned}
$$

For the FE event $\theta_{\text {rms }} \sim 0.14^{\circ} \cdot \mathrm{Z}$, and even for the iron nucleus $(\mathrm{Z}=26)$ deviation is less than $4^{\circ}$.
For the random component of the Galactic magnetic field with an amplitude $B_{\mathrm{rms}} \sim 10^{-6} \mathrm{G}$ and coherence length $l=100 \mathrm{pc}$ the trajectory deviation at the distance $d$ is equal to

$$
\theta \sim 0.3^{\circ} \mathrm{Z} \cdot\left(\frac{d}{10 \mathrm{kpc}}\right)^{\frac{1}{2}}\left(\frac{E}{10^{20} \mathrm{eV}}\right)^{-1},
$$

and even in the case of the iron nuclei does not exceed 2-5 degrees, depending on the amplitude of the magnetic field.

To summarise, the combined effect of the random Galactic and extragalactic magnetic fields can be described as a spreading with respect to the direction to the source by up to $6^{\circ}$ (see also $[1,2,14,19,20]$ ).

The results of the FE event trajectory reconstruction in the regular Galactic magnetic field are presented in Fig. 1 and 2. Fig. 1 shows the recovered position of a potential nearby $(<30 \mathrm{Mpc})$ extragalactic parent source of the FE event on the sky map. The calculations made for proton $(Z=1)$ and iron nuclei $(Z=26)$ showed that the regular Galactic magnetic field in the FE event arrival direction (which corresponds nearly to the Galactic anticentre) decreases mainly over the Galactic latitude (bcoordinate), which results in deflection at an angle of $0.35^{\circ} \cdot \mathrm{Z}$ - mainly due to the deflection in regular magnetic fields of the spiral arms (Fig. 2). The resulting angular bias for a proton $-0.35^{\circ}-$ is still within the error of the initially determined position and therefore is not presented in Fig. 1. For the $\mathrm{C}-\mathrm{N}-\mathrm{O}$ group particles the bias is approximately $2-3$ degrees. The corresponding bias for iron is presented for two values of the CR energy: the measured $\left(9^{\circ}\right)$ and reduced by $1 \sigma\left(11^{\circ}\right)$.

\section{SEARCH FOR THE POTENTIAL SOURCES}

The FE event is far enough from the Galactic plane and within the $5^{\circ}$-circle around its position (the expected deviation of EHECR in random Galactic and extragalactic magnetic fields discussed above) there are no Galactic sources which might be responsible for its origin [18]. Moreover, even in the case of the extragalactic sources the nearest potential standard (blazar) sources of EHECR are much farther $[18,21]$.

In Table 1 we show a detailed list of the potential extragalactic sources of the FE event at the distances up to $30 \mathrm{Mpc}$, that are no more than $8^{\circ}$ from the reconstructed extragalactic position of the event. There are only nine of such galaxies and all of them are not active. Their characteristics (name, type, size (diameter), galactic coordinates $l$ and $b$, separation from calculated source position $\left(l=165.5^{\circ}, b=18.6^{\circ}\right)$, redshift and distance) are presented in the columns 2-9 of Table 1. For an iron nucleus as the UHECR particle, the main source candidate is the galaxy UGC 03574 because of its practical coincidence with the corresponding error box 
(within the discussed above error in 5 overlapping with the corresponding error box (extended by the discussed above $6^{\circ}$ spreading in random magnetic fields, Fig. 1), its large size compared to the other candidates and therefore a larger number of potential UHECR sources (e.g. young pulsars, magnetars, tidal disruption events). For a nucleus from the $\mathrm{C}$ $\mathrm{N}-\mathrm{O}$ - group $(\mathrm{Z}=6-8)$ the magnetic deviation error box is of order of $0.35^{\circ} \cdot Z \sim 2.5^{\circ}$ and the best candidate is the galaxy UGC 03394. It is of smaller size in comparison with UGC 03574, but its advantage is that the $\mathrm{C}-\mathrm{N}-\mathrm{O}$-nucleus as the UHECR particle is in accordance with the recent data about the $\mathrm{CR}$ composition at the highest energies [3, 4, 25]. The position of these and some other galaxies from Table 1 are also shown in Fig. 1.

\section{DISCUSSION AND CONCLUSIONS}

In the reconstructed FE event area there are no suitable galactic candidates and, moreover, there are no nearby AGNs either [18]. Therefore we have performed a search among the ordinary galaxies, in which millisecond pulsars and magnetars together with the tidal disruption of stars in the gravitational fields of the central supermassive black holes are the viable sources of EHECR. The supernovae in these galaxies can give birth to millisecond pulsars or magnetars - the neutron stars with enormous magnetic fields of the order of $10^{15} \mathrm{G}$, which appear as anomalous X-ray pulsars (AXPs) or soft gamma repeaters (SGRs) [13, 15, 21]. Activity of compact objects in our Galaxy provides evidences in favour of this assumption [22]. The main potential sources of the FE event are the galaxy UGC 03574 (in case of Fe nucleus) and the galaxy UGC 03394 (in case of $\mathrm{C}-\mathrm{N}-\mathrm{O}$ nuclei).

The important point for the interpretation of observations is the expected transient nature of the EHECR acceleration in potential sources, as the acceleration occurs during their flaring activity. It may be realised in a form of an explosive activity of compact stars (like the increase of power of relativistic jets or the release of magnetised plasmoids in microquasars, magnetar magnetospheric flares, strong wind activity of newly-born millisecond pulsars) or via the formation of short-lived relativistic jets as a result of the tidal disruption of stars in the gravitational field of a black hole [21]. Such sources of activity must be accompanied by the flares of radiation in different bands of the electromagnetic spectrum (though one should take into account a time delay between the EHECR and photon arrival due to the non-straight-line trajectory in the magnetic field, which in our case is tens to hundreds of years). It is therefore important to have the multi-wavelength observations of the potential candidates for additional verification of the source activities over the right pe- riod of time.

Small statistics of the detected UHECRs, imperfection of the existing Galactic and extragalactic magnetic field models, local inhomogeneity of the extragalactic magnetic field distribution in the vicinity of the our Galaxy impede the search for Galactic and extragalactic sources of the UHECR. Nevertheless, the use of the data on CR of the highest detected energy to search for the CR sources is the most promising approach since EHECR experience the smallest deviations in magnetic fields. Furthermore, the EHECR sources must be situated close enough (up to $50-70 \mathrm{Mpc}$ ) and their transient acceleration process should be accompanied with the bright enough electromagnetic radiation. Thereby the improving statistics of EHECR, especially with the commissioning in near future of the orbital UHECR detector JEM-EUSO, gives hope for the solution of the puzzle of the UHECR sources.

\section{ACKNOWLEDGMENT}

The data in Table 1 are taken from the NASA/IPAC Extragalactic Database ${ }^{1}$. This publication is based on the research supported by the grant of the State Fund For Fundamental Research of Ukraine (project F64/42-2016).

\section{REFERENCES}

[1] Aab A., Abreu P., Aglietta M. et al. 2014, ApJ, 794, 172

[2] Aab A., Abreu P., Aglietta M. et al. 2015, ApJ, 804, 15

[3] Abbasi R. U., Abe M., Abu-Zayyad T. et al. 2014, ApJ, $790, \mathrm{~L} 21$

[4] Abbasi R., BellidoJ., BelzJ. et al. 2014, in 'Proc. UHECR2014, 010016

[5] Albuquerque I. F. M. \& Chou A. 2010, JCAP, 08, 016

[6] Allard D. 2012, Astroparticle Phys., 39, 33

[7] Aloisio R. \& Tortorici F. 2008, Astroparticle Phys., 29, 307

[8] Berezinsky V., Gazizov A. \& Grigorieva S. 2006, Phys. Rev. D, 74, 043005

[9] Bhattacharjee P. \& Sigl G. 2000, Phys. Rep., 327, 109

[10] Bird D. J., Corbato S. C., Dai H. Y. et al. 1995, ApJ, 441, 144

[11] Bleve C. for the Pierre Auger Collaboration. 2015, in 'Proc. ICRC 2015', 61, [arXiv:1509.03732]

[12] Dermer C.D., RazzaqueS., FinkeJ. D. \& Atoyan A. 2009, New J. Phys., 11, 065016

[13] Duncan R. C. \& Thompson C. 1992, ApJ, 392, L9

[14] Durrer R. \& Neronov A. 2013, A\&A Rev., 21, 62

[15] Eichler D. 2005, [arXiv:astro-ph/0504452]

[16] Elbert J. W. \& Sommers P. 1995, ApJ, 441, 151

[17] Epele L. N. \& Roulet E. 1998, Phys. Rev. Lett., 81, 3295

[18] Gnatyk R. B., 2016, Kinematics and Physics of Celestial Bodies, 32, 1, 1

[19] Jansson R. \& Farrar G. R. 2012, ApJ, 757, 14

[20] Jansson R. \& Farrar G. R. 2012, ApJ, 761, L11

[21] Kotera K. \& Olinto A. V. 2011, ARA\&A, 49, 119

${ }^{1}$ http: //ned.ipac.caltech.edu/ 

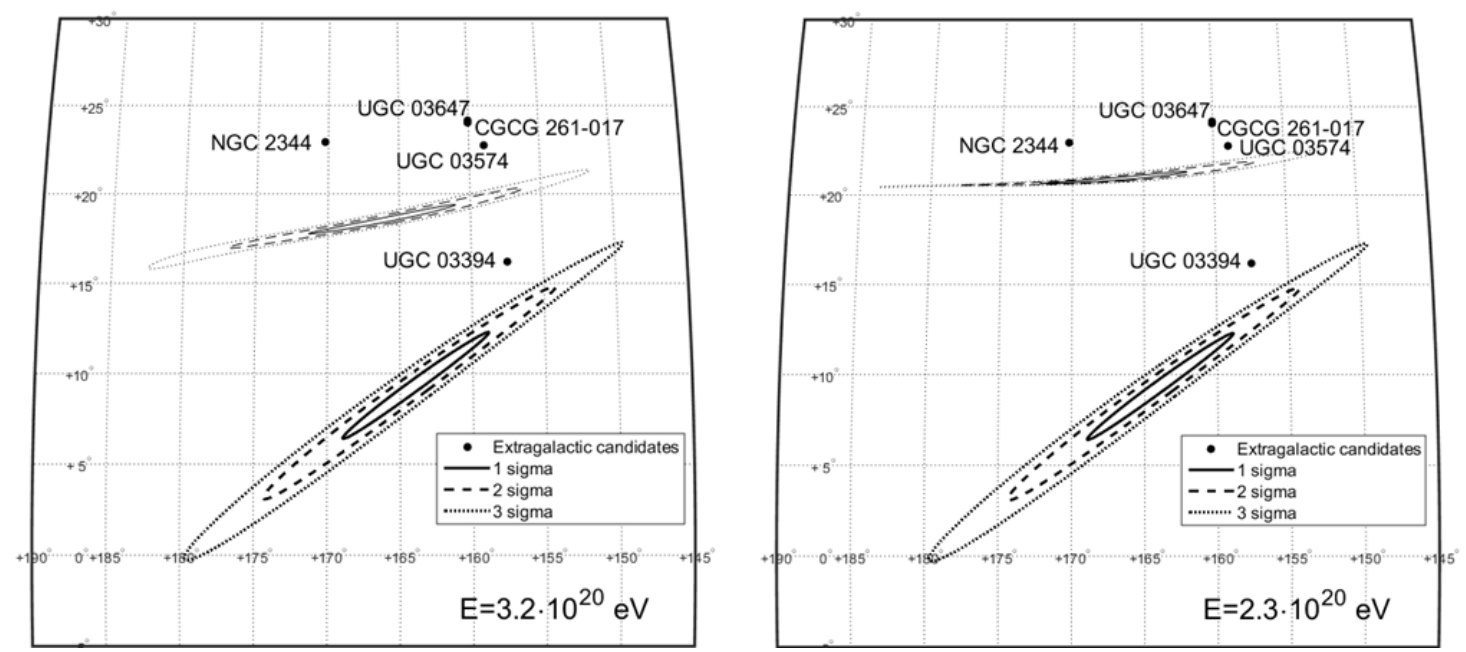

Fig. 1: Sky map with the arrival direction of the FE event (thick lines) and the calculated position of a potential source at the distance of $30 \mathrm{Mpc}$ (thin lines) for the iron nucleus $(Z=26)$ with $E=3.2 \cdot 10^{20} \mathrm{eV}$ (left) and the same for its energy reduced by $1 \sigma E=2.3 \cdot 10^{20} \mathrm{eV}$ (right). The possible extragalactic sources of the FE event are also shown. The galactic coordinates are used.

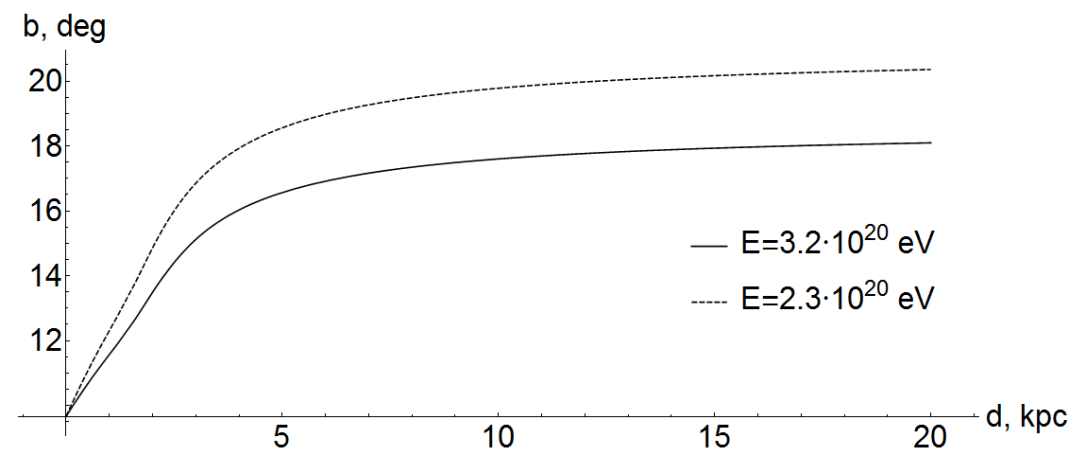

Fig. 2: The increase of the Galactic latitude of the UHECR depicted in Fig. 1 due to its deflection in the regular Galactic magnetic field as a function of the distance travelled. The outer edge of the Galaxy in the $\mathrm{FE}$ event direction is at the distance of $12 \mathrm{kpc}$.

Table 1: Potential candidates of the FE event.

\begin{tabular}{c|l|c|c|l|c|c|c|c}
\hline$\#$ & \multicolumn{1}{|c|}{ name } & $\mathrm{l}^{\circ}$ & $\mathrm{b}^{\circ}$ & \multicolumn{1}{c|}{ Type } & Size, kpc & Separation $\phi, \mathrm{min}$ & $\mathrm{z}$ & $\mathrm{d}, \mathrm{Mpc}$ \\
\hline 1 & UGC 03501 & 166.26 & 18.24 & G Im & 2.3 & 44.6 & 0.00149 & 6.2 \\
2 & kkh 038 & 168.54 & 19.09 & G Ir & 2.5 & 173.3 & 0.0015 & 6.2 \\
3 & NGC 2344 & 170.25 & 22.95 & G SA(rs) c & 7.2 & 372.9 & 0.00325 & 13.3 \\
4 & UGC 03574 & 158.93 & 22.76 & G SA(s) cd & 24.9 & 448.6 & 0.00481 & 19.7 \\
5 & CGCG 261-017 & 160.06 & 24.02 & G & 5.6 & 448.8 & 0.00471 & 19.2 \\
6 & UGC 03647 & 160.05 & 24.14 & G IBm & 8.1 & 454.3 & 0.00462 & 19 \\
7 & UGC 03698 & 172.97 & 21.62 & G Im & 2.1 & 456.9 & 0.00141 & 5.8 \\
8 & NGC 2337 & 172.94 & 21.80 & G IBm & 4.7 & 459.7 & 0.00145 & 6.0 \\
9 & UGC 03394 & 157.53 & 16.18 & G SB? & 13.1 & 480.6 & 0.00607 & 24.9 \\
\hline
\end{tabular}

[22] Olausen S. A. \& Kaspi V. M. 2014, ApJ Suppl., 212, 6

[23] Stecker F. W. \& Salamon M. H. 1999, ApJ, 512, 521

[24] Takami H., Murase K. \& Dermer C. D. 2016, ApJ, 817,
59

[25] Yushkov A.,for the Pierre Auger Collaboration. 2015, in 'Proc. ICRC 2015', 47, [arXiv:1509.03732] 